\title{
Estimation of Stability Derivatives for a Planar Wedge in the Newtonian Limit
}

\author{
Asha Crasta ${ }^{1}$ and S. A. Khan ${ }^{2}$ \\ ${ }^{1}$ Research Scholar, Department of Mathematics, Jain University, Bangalore, Karnataka, India, \\ ${ }^{2}$ Principal, Mechanical Engineering Department, Bearys Institute of Technology, Innoli Mangalore, \\ Karnataka, India,
}

\begin{abstract}
The present work contains an analytical method derived using Ghosh's Hypersonic similitude to predict the aerodynamic stability derivatives of a Planar Wedge in the Newtonian limit. It uses the strip theory developed by Ghosh's where span wise strips are independent of each other, to obtain the expressions for stiffness and damping derivatives in pitch for a planar wedge in the Newtonian limit. The present theory predicts the stability derivatives of a planar wedge for a wide range of geometrical and flow parameters. The knowledge of these stability derivatives is essential to freeze and arrive at the geometrical as well as the kinematic similarity parameters before we go for exhaustive computations and experimental studies. The present method predicts the stability derivatives in pitch for a planar wedge with remarkable computational ease, which is very handy at the design stage. The expressions derived for stability derivatives become exact in the Newtonian limit. It is found that stiffness derivative linearly varies with the pivot position.

It is also observed that the centre of pressure moves towards the trailing edge and this shift is quite high at high angles of attack. Hence, this behavior could be utilized to stabilize the aerospace vehicle from the static stability point of view. In the case of damping derivative since the expression for the damping derivative is non-linear and the same has been reflected in the results. However, the behavior remains linear till angle of attack fifteen degrees, later the trend is non-linear.
\end{abstract}

Keywords: angle of incidence, Newtonian Limit, Strip theory, Planar wedge, pivot position

\section{Introduction:}

Unsteady supersonic/hypersonic aerodynamics has been studied extensively for moderate Supersonic Mach number and hypersonic Mach number for small angles of attack only and hence there is evidently a need for a unified supersonic/hypersonic flow theory that is applicable for large as well as small angles of attack.

For two-dimensional flow, exact solutions were given by Carrier [1] and Hui [2] for the case of an oscillating wedge and by Hui [3] for an oscillating flat plate. They are valid uniformly for all supersonic Mach numbers and for arbitrary angles of attack or wedge angles, provided that the shock waves are attached to the leading edge of the body.

For an oscillating triangular wing in supersonic/hypersonic flow, the shock wave may be attached or detached from the leading edges, depending on the combination of flight Mach number, the angle of attack, the ratio of specific heats of the gas, and the swept-back angle of the wing. The attached shock case was studied by Liu and Hui [4] where as the detached shock case in hypersonic flow was studied by Hui and Hemdan both are valid for moderate angles of attack. Hui et.al [5] applied the strip theory to study the problem of stability of an oscillating flat plate wing of arbitrary plan form shape placed at a certain mean angle of attack in a supersonic/hypersonic stream. The strip theory becomes exact in the Newtonian limit since the Newtonian flow, in which fluid particles do not interact with each other is truly two-dimensional locally. Crasta and Khan have extended the Ghosh theory [6] to planar wedges in Hypersonic and Supersonic Flow([10]\&[11]). Further Crasta and Khan have extended this theory for Delta wings with Straight leading edges ([7] \&[8])and curved leading edges([12]\&[13]) in Hypersonic and Supersonic Flows. The stability derivatives in Newtonian limit for Straight leading [9] and curved leading edges[14] have also been studied by Crasta and Khan. In this paper Hypersonic Planar similitude is been used and the relations have been obtained for the stability derivatives in pitch and roll in the Newtonian limit for a planar wedge. 
Consider a Planar wedge as shown in fig

\section{ANALysis}

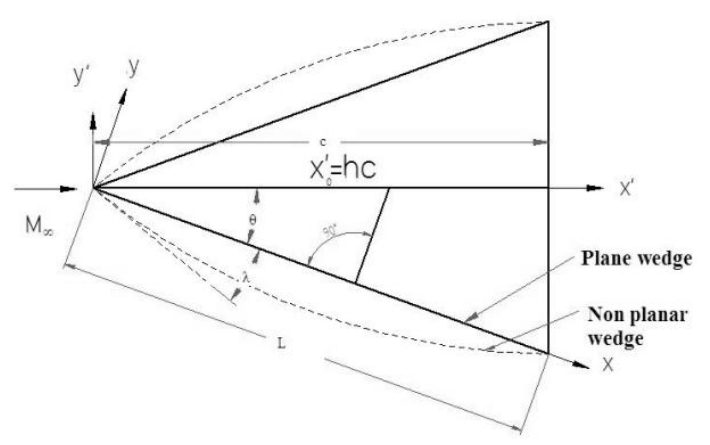

Fig. A. Planar wedge transfer of pivot position from $x_{\circ}$ to $x_{\circ}^{\prime}$

The Stiffness and damping derivative is given by

$$
-C_{m_{\alpha}}=(\gamma+1)\left(\tan \alpha_{0}\right)\left(2+D+\frac{1}{D}\right)\left(\frac{1}{2}-h \cos ^{2} \alpha_{0}\right)
$$

(1)

$$
-C_{m_{q}}=(\gamma+1)\left(\frac{\tan \alpha_{0}}{\cos ^{2} \alpha_{0}}\right)\left(2+D+\frac{1}{D}\right)\left(\frac{1}{3}-h \cos ^{2} \alpha_{0}+h^{2} \cos ^{4} \alpha_{0}\right)
$$

$$
\text { where } D=\frac{\sqrt{\left(\frac{4}{\gamma+1}\right)^{2}+M_{\infty}^{2} \sin ^{2} \alpha_{0}}}{M_{\infty} \sin \alpha_{0}}
$$

In the Newtonian limit $M_{\infty}$ tends to infinity and $\gamma$ tends to unity.

$\lim _{\substack{M_{\infty} \rightarrow \infty \\ \gamma \rightarrow 1}} D=\lim _{\substack{M_{\infty} \rightarrow \infty \\ \gamma \rightarrow 1}} \frac{\sqrt{\left(\frac{4}{\gamma+1}\right)^{2}+M_{\infty}^{2} \sin ^{2} \alpha_{0}}}{M_{\infty} \sin \alpha_{0}}=1$

(3)

$$
\lim _{\substack{M_{\infty} \rightarrow \infty \\ \gamma \rightarrow 1}}(\gamma+1)\left(2+D+\frac{1}{D}\right)=8
$$

Therefore the Stiffness and Damping derivatives in Newtonian limit is given by

$$
-C_{m_{\alpha}}=4 \tan \alpha_{0}-8 \mathrm{~h} \tan \alpha_{0} \cos ^{2} \alpha_{0}
$$

$$
-C_{m_{q}}=\frac{8 \tan \alpha_{0}}{\cos ^{2} \alpha_{0}}\left(\frac{1}{3}-h \cos ^{2} \alpha_{0}+h^{2} \cos ^{4} \alpha_{0}\right)
$$

We define $g(h)=\left(\frac{1}{3}-h \cos ^{2} \alpha_{0}+h^{2} \cos ^{4} \alpha_{0}\right)$ which is a quadratic in pivot position $\mathrm{h}$ and hence has a minimum value

$$
-C_{m_{q}}=\frac{8 \tan \alpha_{o} g(h)}{\left(\cos ^{2} \alpha_{o}\right)}
$$


In Eq. (6), only g (h) depends on $\mathrm{h}$ and other terms are constant. To get minimum value of $C_{m_{q}}$ only $\mathrm{g}(\mathrm{h})$ is to be differentiated and putting $\frac{\partial}{\partial h} g(h)$ equal to zero

$$
\frac{\partial}{\partial h}\left[\left(\frac{1}{3}-h \cos ^{2} \alpha_{0}+h^{2} \cos ^{4} \alpha_{0}\right)\right]=0
$$$$
\therefore h=\frac{1}{2 \cos ^{2} \alpha_{0}}
$$

Let the value for h corresponding to $\left[C_{m q}\right]_{\min }$ be denoted $h_{m}$.

$$
\therefore h_{m}=\frac{1}{2 \cos ^{2} \alpha_{0}}
$$

Hence $g(h)_{\min }=\left(\frac{1}{3}-h_{m} \cos ^{2} \alpha_{0}+h_{m}{ }^{2} \cos ^{4} \alpha_{0}\right)$

$$
\therefore-C_{m_{q}}=\frac{8 \tan \alpha_{o} g(h)_{\min }}{\left(\cos ^{2} \alpha_{o}\right)}
$$

\section{Results And Discussions}

The present study was taken up to derive the analytical expressions for stiffness and damping derivative in pitch for a planar wedge in supersonic/hypersonic flow. Later, the same study was extended to obtain the closed form solutions for stiffness and damping derivatives in pitch for a planar wedge in the Newtonian limit; where, the Mach number will tend to infinity and the Specific heat ratio gamma $(\gamma)$ will tend to one, in this situation when the stability derivatives are considered how they will vary with the change in the geometric parameters as well as the flow parameters.

Fig. 1 shows the variation of stiffness derivatives with the non-dimensional pivot position. From the figure it is seen that for various angle of incidences the stiffness derivatives decreases linearly with pivot position and the trend is on the expected lines. Further, it is seen that the stiffness derivative linearly increases with the increase of angle of attack which is very true and this trend was expected. From the figure it is seen that there is gradual increase in the value of the stiffness derivatives. When the angle of attack has increased from five degrees to ten degrees there is a forty eight percent increase in the stiffness derivative. Similarly, for the range between ten degrees to fifteen degrees the increase in the stiffness derivatives is around fifty percent. Further, it is found that for range between fifteen degrees to twenty degrees there is a fifty six percent increase in the stiffness derivative. Similarly, for the angle of incidence in the range twenty to twenty five and twenty five to thirty degrees the increase in the stiffness derivative is observed to be in the range sixty two to sixty five percent.

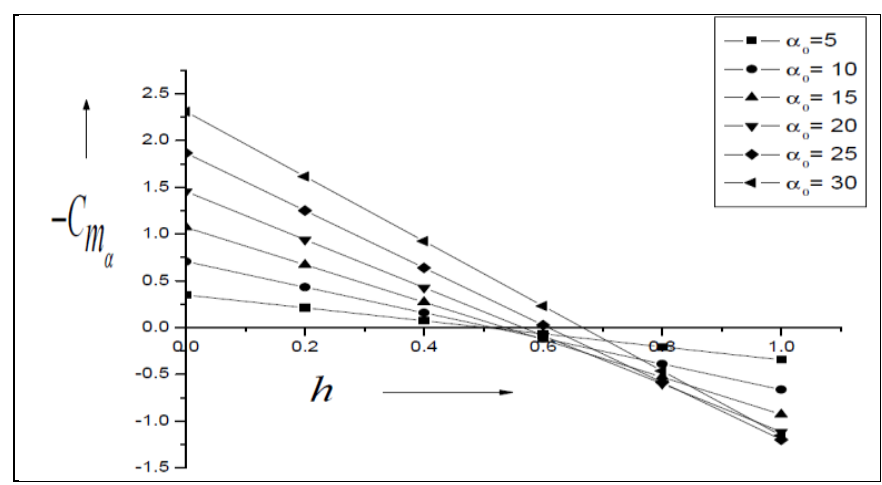

Fig. 1: variation of stiffness derivative with pivot position in the Newtonian limit

From the figure another observation is that with increase in the angle of attack there is a continuous shift of the center of pressure towards the aft position of the wedge. Due to this continuous shift of the center of pressure, this method of continuous increasing the angle of incidence could be used to stabilize the aerodynamic vehicles and the requirement of large stabilizing surface could be avoided or in some cases the use of the tail fins can be completely avoided. 
Results for damping derivatives are shown in Fig. 2 for various angles of incidence from five degrees up to thirty degrees. From these results it is evident that with the increase in angle of attack at $h=0.0$ there is a continuous increase in the values of the damping derivatives, it is also observed that for lower angles of incidence the curve becomes flat for the pivot positions in the range from $\mathrm{h}=0.3$ to 0.6 , and this trend may be due to the variation in the pressure distribution on the non-planar surface. It is found that for angle of attack in the range five degrees to ten degrees, ten degrees to fifteen degrees, fifteen degrees to twenty degrees, twenty degrees to twenty five degrees, and twenty five degrees to thirty degrees the increase in the damping derivatives are hundred percent, fifty percent, forty eight percent, thirty five percent, forty three percent, respectively. Also, for the same range of angle of attack the range for the band of minimum damping derivates are 0.3 to $0.75,0.4$ to $0.6,0.5$ to $0.65,0.52$ to $0.68,0.58$ to 0.68 , and 0.62 to 0.72 ; respectively. We need to have a closer look at these values from the static and dynamic stability point of view. From these results it is evident that these results show a strong sign for future research in this area as well as utility of the data for the further studies.

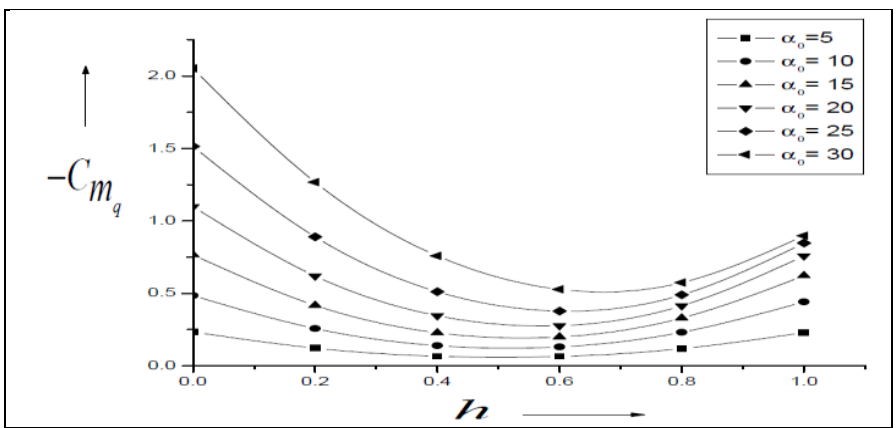

Fig. 2: variation of damping derivative with pivot position in the Newtonian Limit.

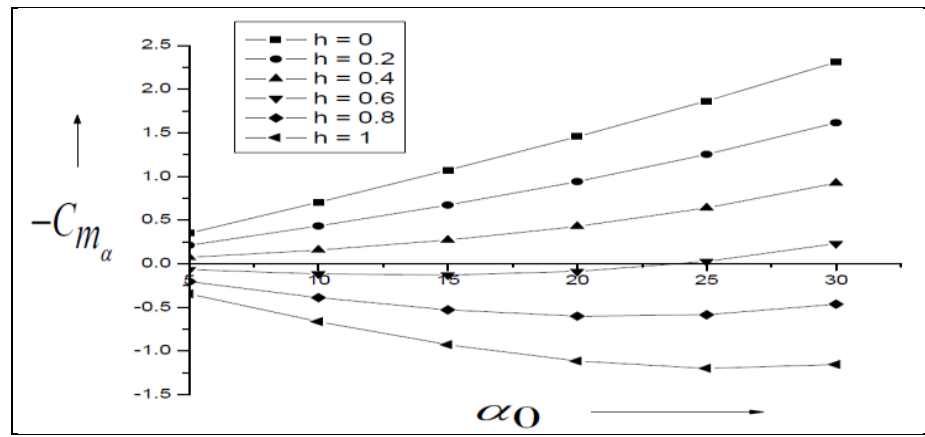

Fig. 3: variation of stiffness derivative with angle of incidence in the Newtonian Limit.

Results of Stiffness derivative as a function of angle of attack are shown in Fig. 3, as a function of for a fixed pivot position of $\mathrm{h}=0, \mathrm{~h}=0.2, \mathrm{~h}=0.4, \mathrm{~h}=0.6, \mathrm{~h}=0.8$, and $\mathrm{h}=1.0$. From the figure it is seen that the Stiffness derivative increases linearly with angle of incidence. It is seen that the stiffness derivative has higher value at $\mathrm{h}=0.0$ when compared to that when it is computed at the points in the downstream towards the trailing edge. It is seen that the non-linearity has crept in the results for $h=0.6,0.8$, and 1.0 . The reasons for this trends could be due to the variations of the center of pressure with angle of attack with the reference to the high angle of attack namely fifteen degrees and above. It is well known that whenever there is an increase in the angle of attack the center of pressure will keep on shifting towards the trailing edge.

Results for damping derivatives as a function of angle of attack are shown in Figs. 4 \& 5. Fig. 4 presents the damping derivative results with respect to angle of attack for $h=0,0.2,0.4$, and 0.6 , whereas, Fig. 5 presents the damping derivatives results for $\mathrm{h}=0.8$ and $\mathrm{h}=1$. It is seen that damping derivatives increases linearly with angle of attack up to fifteen degrees and then for angle of attack greater than fifteen there is change in the trend, and it becomes non-linear. This non-linear variation of the damping derivative is due to the combined effect of angle of attack, pressure distribution at the surface of the wedge and the flow dynamics. It is also seen that the maximum value is achieved for $h=0,0.2$, and 0.8 for all the values of angle of incidence of the present study. 


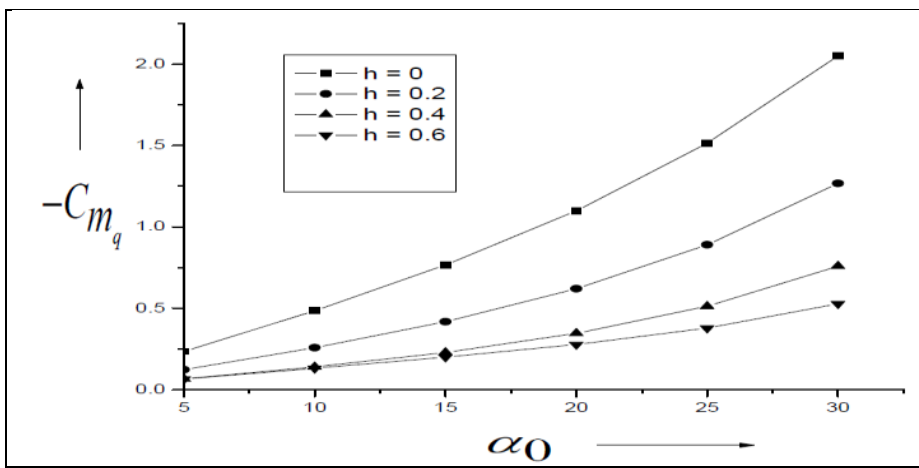

Fig. 4: variation of damping derivative with angle of incidence in the Newtonian limit.

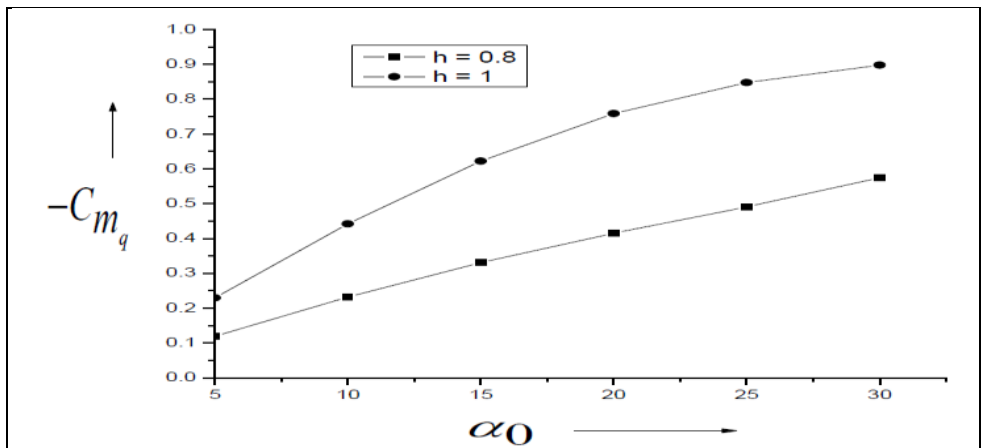

Fig 5: variation of damping derivative with angle of incidence in the Newtonian limit.

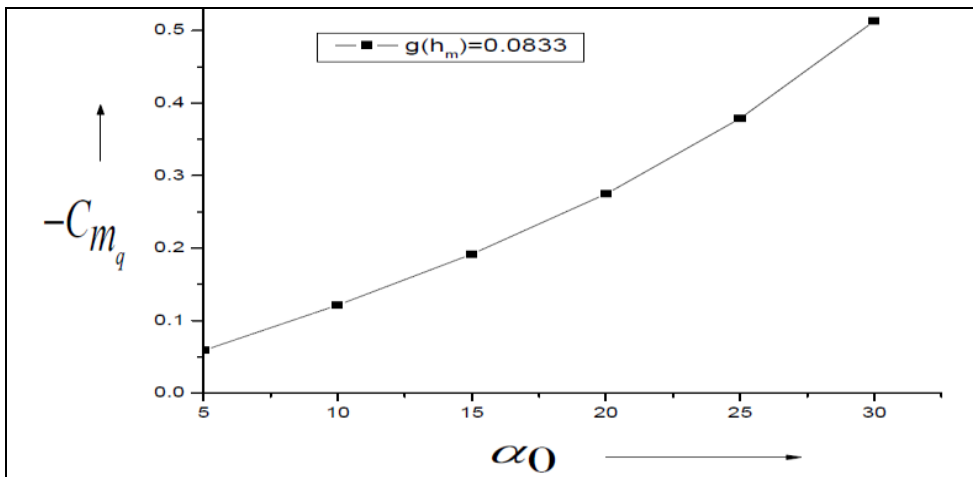

Fig 6: Variation of damping derivative with angle of incidence at minimum pivot position

Fig. 6 presents the results for damping derivative in pitch with angle of attack for a pivot position which will result in minimum damping value, it seen that for this case too, the behavior of the damping derivative remains linear, and increases with angle of attack up to fifteen degrees then it becomes non-linear. The reason for this trend may be the same as discussed above.

\section{Conclusion}

The present theory is valid when the shock wave is attached to the leading edge. The effect of secondary wave reflections and viscous effects are neglected. The expressions derived for stability derivatives become exact in the Newtonian limit. From the results it is found that the stability derivatives are independent of Mach number as they are estimated in the Newtonian limit where Mach numbers will tend to infinity and specific heat ratio gamma will tend to unity. It is found that stiffness derivative linearly varies with the pivot position as the same was found for the cases in our previous results at low supersonic, supersonic, and Hypersonic Mach numbers. Due to the increase in angle of attack the stiffness derivative increases linearly for the entire range of the present study. It is also observed that the centre of pressure moves towards the trailing edge and this shift is quite high at high angles of attack. Hence, this behavior could be utilized to stabilize the aerospace vehicle from the static stability point of view. In the case of damping derivative since the expression for the damping derivative is non-linear and the same has been reflected in the results. However, the behavior remains linear till angle of attack fifteen degrees, later the trend is non-linear. 


\section{References}

[1] Carrier G. F., The Oscillating Wedge in a Supersonic Stream, Journal of the Aeronautical Sciences, Vol.16, March 1949, pp.150152.

[2] Hui W. H., Stability of Oscillating Wedges and Caret Wings in Hypersonic and Supersonic Flows, AIAA Journal, Vol.7, August 1969, pp. 1524-1530.

[3] Hui. W. H., Supersonic/Hypersonic Flow past an Oscillating Flat plate at Large angles of attack, Journal of Applied Mathematics and Physics, Vol. 29, 1978, pp. 414-427.

[4] Liu D. D and Hui W. H., Oscillating Delta Wings with attached Shock waves, AIAA Journal, Vol. 15, June 1977, pp. 804-812.

[5] Hui W. H. et al, Oscillating Supersonic/Hypersonic wings at High Incidence, AIAA Journal, Vol. 20, No.3, March1982, pp. 299304.

[6] Ghosh, K and Mistry B. K., Large incidence hypersonic similitude and oscillating non-planar wedges, AIAA Journal, August 1980, 18,8 , pp. 1004-1006

[7] Asha Crasta and Khan S. A, Oscillating Supersonic delta wing with Straight Leading Edges, International Journal of Computational Engineering Research, Vol. 2, Issue 5, September 2012, pp.1226-1233,ISSN:2250-3005.

[8] Asha Crasta, M. Baig and S. A. Khan, Estimation of Stability derivatives of a Delta wing in hypersonic flow, International Journal of Emerging trends in Engineering and Developments in Vol. 6, Issue 2, Sep2012, pp. 505-516, ISSN: $2249-6149$.

[9] Asha Crasta and Khan S. A., Stability derivatives of a Delta Wing with Straight leading edge in the Newtonian Limit, International Journal of Engineering research and Applications, Vol. 4, Issue 3(Version2), March 2014, pp. 32-38.

[10] ASHA CRASTA and S. A. KHAN , "Hypersonic Similitude for Planar Wedges " in the International Journal of Advanced Research in Engineering and Technology,Volume:5,Issue:2, Feb 2014,pp .16-31.

[11] Asha Crasta and Khan S. A., High Incidence Supersonic similitude for Planar wedge, International Journal of Engineering research and Applications, Vol. 2, Issue 5, September-October 2012, pp. 468-471, ISSN: 2248-9622,Impact Factor 1.324

[12] Asha Crasta and S. A. Khan, Estimation of stability derivatives of Oscillating Hypersonic delta wings with curved leading edges, International Journal of Mechanical Engineering \& Technology, vol. 3, Issue 3, Dec 2012, pp. 483-492. ISSN 0976 - 6340.

[13] [13] S.A Khan, Asha crasta, 'Oscillating supersonic delta wings with curved leading edges', in international journal of Advanced studies in Contemporary Mathematics. Vol 20 , No.3 July 2010, PP 359-372.

[14] Asha Crasta and S. A. Khan, Stability Derivatives in the Newtonian Limit, The International Journal of Advanced Research in Engineering and Technology,Volume:4,Issue:7, Nov-Dec 2013, pp. 276-289. ISSN 0976 - 6480. 